\title{
Matrix Softening Induces Inflammatory Signals of Endothelial Cells
}

\author{
Weicong Chen1, Jiaqi Liang1, Baoxiang Tian1, Yue Zhou' ${ }^{*}$, Song Li $^{2}$ \\ ${ }^{1}$ School of Biomedical Engineering and Med-X Research Institution, Shanghai Jiao Tong University, Shanghai, China \\ ${ }^{2}$ Department of Bioengineering and Department of Medicine, University of California, Los Angeles, CA, USA \\ Email: *yzhou2009@sjtu.edu.cn
}

How to cite this paper: Chen, W.C., Liang, J.Q., Tian, B.X., Zhou, Y. and Li, S. (2019) Matrix Softening Induces Inflammatory Signals of Endothelial Cells. Journal of Biosciences and Medicines, 7, 137-145. https://doi.org/10.4236/jbm.2019.711012

Received: March 4, 2019

Accepted: November 24, 2019

Published: November 27, 2019

\begin{abstract}
Vascular diseases such as atherosclerosis involve the change of the rigidity in the blood vessel wall. There is evidence that the changes in the blood vessel rigidity may affect the various functions of the cells in the blood vessel, including endothelial cells (ECs) and the smooth muscle cells. On the other hand, blood vessel-on-a-chip has become an emerging research field for disease modeling. However, the effect of material rigidity on blood vessel remodeling is not well understood. Hereby, an in vitro culture system with the culture substrates matching the rigidity of vessel wall mimicking the condition of healthy (normal) or lipid deposition (soft) were prepared. The stiffness of the substrates was confirmed by atomic force microscope. Although no significant difference was observed in EC morphology, the expression levels of the pro-inflammatory cytokines, including interleukin 6 (IL6), tumor necrosis factor $\alpha$ (TNF- $\alpha$ ) and interleukin $1 \beta$ (IL1 $\beta)$, were dramatically induced by soft substrate. Consistently, the inflammation-related JNK signaling was also activated. In addition, the expression level of microRNA-146a (miR-146a) was significantly decreased. Accordingly, mRNA expression level of TNF receptor associated factor 6 (TRAF6), the direct target of miR-146a, was significantly increased. In summary, these findings provide new insight into the matrix rigidity effect on ECs; while engineering the blood vessel model in vitro, matrix with proper rigidity can be carefully tailor to mimic ECs either in a quiescent or an inflammation state.
\end{abstract}

\section{Keywords}

Endothelial Cells, Inflammatory Response, Rigidity

\section{Introduction}

Atherosclerosis involves chronic inflammation [1]. There is also evidence that 
atherosclerosis [2] is regulated by biophysical factors, including flow pattern, strain stress, surface topography [3] [4] [5] and rigidity. Due to the deposition of low density lipoprotein (LDL) [6], the rigidity of the blood vessel wall may decrease to $5.5 \pm 3.5 \mathrm{kPa}$ [7]. It was reported that increased rigidity induced the change of cytoskeleton and the activation of signaling pathway of cells [8] [9]. However, whether softening impacts the development of atherosclerosis is still poorly understood.

On the other hand, organs-on-a-chip (OOCs) can be used as an in vitro disease model [10]. For the investigation of vascular inflammation, tissue engineering, drug screening and vascularization, several vessel-on-a-chip system were designed [11] [12] [13]. However, these studies mainly focused on the designing of the physical structure of the systems, with fewer consideration of the rigidity of the materials, which is an indispensable influence factor of vascular cell functions.

In this study, substrates matching the rigidity of softened vessel and normal vessel were fabricated, and how substrate rigidity exerts and impacts the inflammatory response and underlying mechanism was investigated.

\section{Materials and Methods}

\subsection{Substrate Preparation and Rigidity Measurement}

Substrates with soft rigidity $(8 \mathrm{kPa})$ and normal rigidity $(20 \mathrm{kPa})$ were prepared following the method mentioned in a previous research [14]. Specifically, solutions with different proportions components, including acrylamide (Sangon Biotech, China) and bis-acrylamide (Sangon Biotech, China), 10\% ammonium persulfate solution and tetramethylethylenediamine (TEMED, Klamar, China) were prepared. Then $160 \mu \mathrm{L}$ solution was dropped onto the gel slick-treated glass slide. A silanized cover glass was then placed onto the top of the liquid quickly. After polymerization, the substrate was removed from the glass slide and coated with collagen I (Corning, USA).

The rigidity of polyacrylamide (PA) substrates was detected by atomic force microscope (AFM, Bruker, Germany). The probe used for the AFM measurement was a glass ball probe with a radius of $5 \mu \mathrm{m}$. Three random points of each substrate were measured and the results were shown as mean \pm SEM.

\subsection{Cell Culture}

Human umbilical vein endothelial cells (HUVECs, Sciencell, USA) were cultured in endothelial cell culture medium (Sciencell, USA), containing penicillin/streptomycin (1\%), endothelial cell growth supplement (1\%) and fetal bovine serum (5\%). HUVECs were culture at $37^{\circ} \mathrm{C}$ and $\mathrm{CO}_{2}(5 \%)$.

\subsection{Cell Imaging and Staining}

HUVECs were cultured on different substrates for 2 hours and imaged with a reverted microscope (Leica, German). When reached confluence, the cells were 
fixed with paraformaldehyde (4\%). FITC-labeled phalloidin was used to stain the cytoskeleton and DAPI was used to stain the nuclei. After mounting, the samples were observed under laser scanning confocal microscope (Leica, Germany). All the processes were conducted in dark place to avoid photo-bleaching.

\subsection{RNA Extraction and RT-qPCR Analysis}

An RNA simple total RNA extraction kit (Tiangen Biotech, China) was used to extract total RNA following the manufacturer's instruction. The genomic DNA was removed by gDNase treatment. After reverse transcription process, the samples were analyzed using a Talent qPCR PreMix kit (Tiangen Biotech, China) by SYBR green method. The internal control used in this study was GAPDH. All mRNA primers were provided by BioTNT (China) and the sequences were shown in Table 1.

A miRcute miR Isolation Kit (Tiangen Biotech, China) was used for extraction of microRNAs. Then the samples were reverse transcribed into complementary DNA (cDNA) using a miRcute miR First-strand cDNA synthesis kit (Tiangen Biotech, China). The cDNA was analyzed by SYBR green method using a miRcute miR qPCR Detection kit (Tiangen Biotech, China) following the instruction of the product. U6 was used a microRNA internal reference for the detection of microRNAs. The catalog numbers of U6 and miR-146a were CD201-0145 and CD201-0013, respectively. All microRNA primers were bought from Tiangen Biotech (China).

\subsection{Western Blot Analysis}

Whole cell lysates were extracted using RIPA buffer with the addition of phosphatase inhibitors and protease inhibitors (Tiangen Biotech, China). After quantification, equal amount of the samples were denaturized and loaded. Then the proteins were separated by SDS-PAGE and transferred into a PVDF membrane (Bio-Rad, USA). After blockage for 1 hour, the samples were incubated with mouse-anti-human p-JNK primary antibody (Cell signaling technology, USA) or rabbit-anti-human JNK primary antibody (Cell signaling technology, USA) at $4^{\circ} \mathrm{C}$ overnight. After rinsed for 3 times, the membranes were incubated with HRP-conjugated rabbit-anti-mouse secondary antibody (Cell signaling technology, USA) or goat-anti-rabbit secondary antibody (Cell signaling technology, USA) for 1 hour at the room temperature. After washing, the samples were detected

Table 1. The sequence of primers used for mRNA real time PCR.

\begin{tabular}{clc}
\hline Targets & \multicolumn{1}{c}{ Forward primer (from 5’-3’) } & \multicolumn{1}{c}{ Reverse primer (from 5’-3’) } \\
\hline GAPDH & GGG AAG GTG AAG GTC GGA GT & GGG GTC ATT GAT GGC AAC A \\
IL6 & GCA CCT CAG ATT GTT GTT G & AAA TAG TGT CCT AAC GCT CA \\
TNF- $\alpha$ & AGT CTG GGC AGG TCT ACT TT & CGT TTG GGA AGG TTG GAT GT \\
IL1 $\beta$ & GCT GGC AGA AAG GGA ACA GA & GCA GTT GGG CAT TGG TGT AG \\
TRAF6 & GCT GTT CAT AGT TTG AGC GT & TCT TAC AAG GCG ACC CTC T \\
\hline
\end{tabular}


using an enhanced chemiluminescence system (Bio-Rad, USA) and semi-quantified with Image $\mathrm{J}$ by comparing the intensity of the images (NIH, USA). $\beta$-tubulin was used as an internal reference in the western blot assay.

\subsection{Statistics}

GraphPad Prism 6.0 software was applied for statistical analysis. All data represented the mean of triplicates unless otherwise indicated and error bars signified standard error of the mean (SEM). Unpaired student's t-test was used for the comparison of two means. Significance was considered if $\mathrm{p}$ value $<0.05$ and marked with *.

\section{Results}

\subsection{Substrate Preparation and Rigidity Measurement}

The rigidity of PA substrates can be tuned by adjusting the proportion of acrylamide and bis-acrylamide. Using AFM, the rigidity of different substrates was confirmed. As Figure 1 indicated, the slope of the curves indicated the rigidity of the matrices. The dotted line showed a more inclined slope than the solid line, indicating the higher rigidity of the $20 \mathrm{kPa}$ than $8 \mathrm{kPa}$. Furthermore, young's modulus of the substrates verified that rigidity of the fabricated substrates was consistent with our expectation. The mean rigidity was $7.37 \mathrm{kPa}$ and $23.2 \mathrm{kPa}$, respectively (Table 2). Thus substrates with rigidity of $8 \mathrm{kPa}$ and $20 \mathrm{kPa}$ were fabricated successfully.

\subsection{Substrate Rigidity Showed No Influence on ECs Morphology}

HUVECs were seeded onto the surface of the collagen I coated PA substrates.

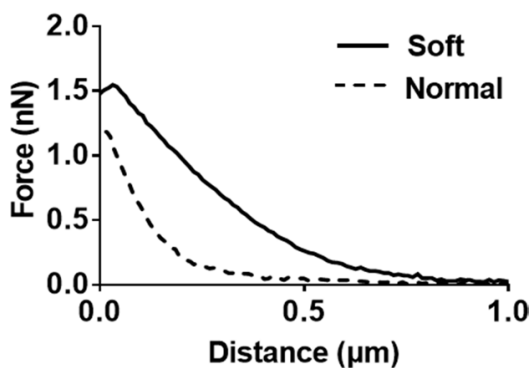

Figure 1. Young's modulus measurement of soft and normal substrates by AFM. The young's modulus (rigidity) of the substrates was measured by atomic force AFM. A glass ball probe with radius of $5 \mu \mathrm{m}$ was used for the measurement. The solid line and dotted line referred to the force-distance of soft substrate and normal substrate, respectively. The slopes of the curves referred to the young's modulus of the substrates.

Table 2. Young's modulus of substrate rigidity by AFM.

\begin{tabular}{ccc}
\hline Expected rigidity $(\mathrm{kPa})$ & Mean rigidity & Standard error \\
\hline 8 & 7.37 & 0.69 \\
20 & 23.2 & 3.40 \\
\hline
\end{tabular}


After 2 hours of incubation, the ECs were imaged. As Figure 2(A) \& Figure 2(B) indicate, ECs already adhered onto the PA substrates and no observable significance was found. After the ECs formed monolayers on PA substrates, the cytoskeleton was stained. However, the polygonal morphology of ECs was observed and no obvious change of morphology was acquired on both soft and normal substrates (Figure 2(C) \& Figure 2(D)).

\subsection{Substrate Rigidity Regulates Expression of Pro-Inflammatory Cytokines}

After ECs reached complete confluence on different substrates, the total RNA was extracted and analyzed. The expression of IL6, TNF- $\alpha$ and IL1 $\beta$ was dramatically increased in ECs on soft substrate compared to normal substrate (Figure 3). Concretely, the expression of IL6, TNF- $\alpha$ and IL1 $\beta$ in ECs on soft substrate was
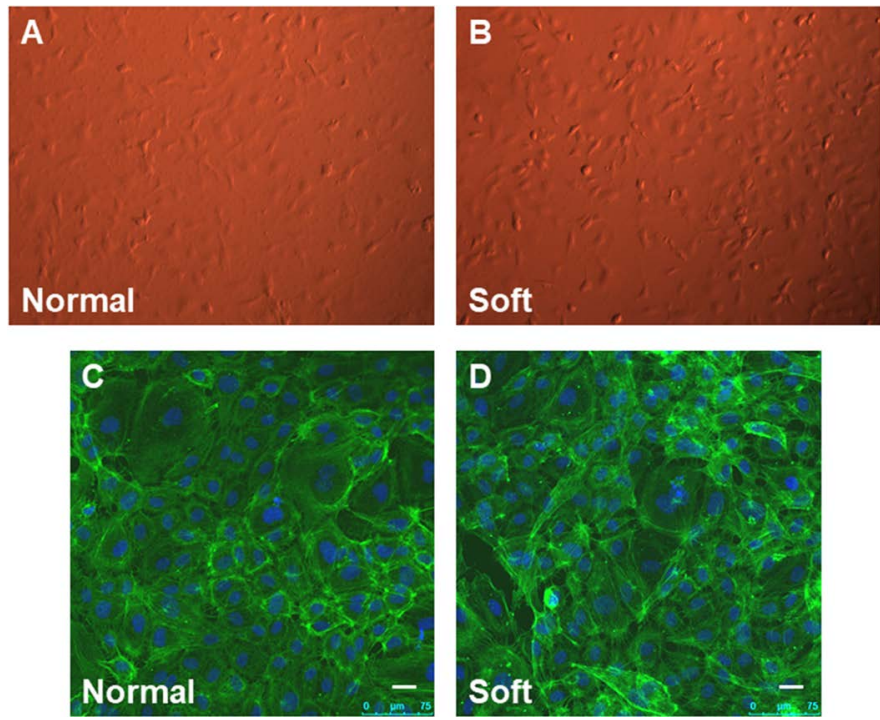

Figure 2. Pictures of ECs acquired with optical microscopes. ECs were seeded on the surface of the substrates and after 2 hours of adhering, the cells were observed by an inverted microscope under bright field ((A) \& (B)). After ECs formed monolayers on substrates, cells were fixed. The cytoskeleton was stained green by FITC-labeled phalloidin and the nuclei were stained blue by DAPI ((C) \& (D)). The scale bar is $25 \mu \mathrm{m}$.
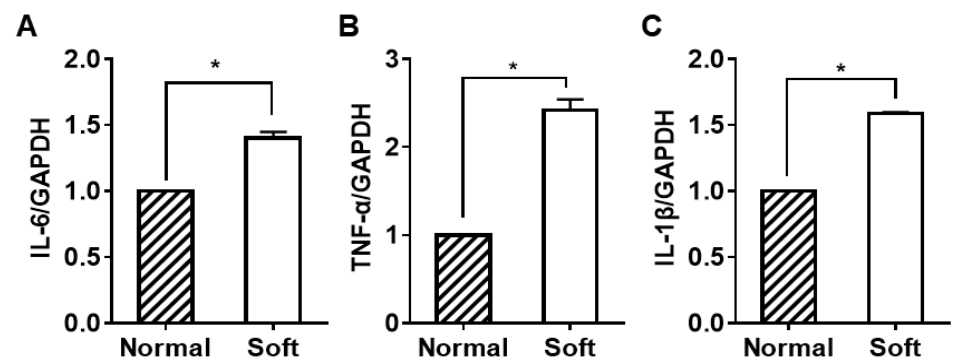

Figure 3. Gene expression of pro-inflammatory cytokines in ECs. After ECs reached complete confluence on substrates, the mRNA expression of interleukin 6 (A), tumor necrosis factor $\alpha(\mathrm{B})$ and interleukin $1 \beta(\mathrm{C})$ were analyzed by RT-qPCR. GAPDH was used as an internal control. 
about 1.4-fold (Figure 3(A)), 2.4-fold (Figure 3(B)) and 1.6-fold (Figure 3(C)) compared with normal substrate. Therefore, these results indicated that soft substrate induced the pro-inflammatory response of ECs.

\subsection{Substrate Rigidity Regulates Inflammatory Signaling Pathways in ECs}

Since the expression of inflammatory cytokines was changed by substrate rigidity, we then studied whether the inflammation-related signaling cascades were impacted by substrate rigidity. C-Jun N-terminal kinases (JNK) in mitogen-activated protein kinase (MAPK) signaling pathway were shown to regulate the expression of inflammatory cytokines, including IL6, TNF- $\alpha$ and IL1 $\beta$ [15] [16]. Thus, the activation of MAPK signaling pathway was analyzed (Figure 4). Unexpectedly, the phosphorylation of JNK in ECs on soft substrate was significantly enhanced compared to normal substrate (Figure 4(A) and Figure 4(B)), illustrating strong regulation of JNK signaling pathway by ECM rigidity.

To further explore the reason why JNK signaling pathway was involved in EC inflammatory response induced by substrate rigidity, the expression of TNF receptor associated factor 6 (TRAF6), an important regulator of JNK signaling path way, was examined. Surprisingly, the expression of TRAF6 in soft rigidity group was high than normal rigidity group (Figure 5(A)), implying activation of
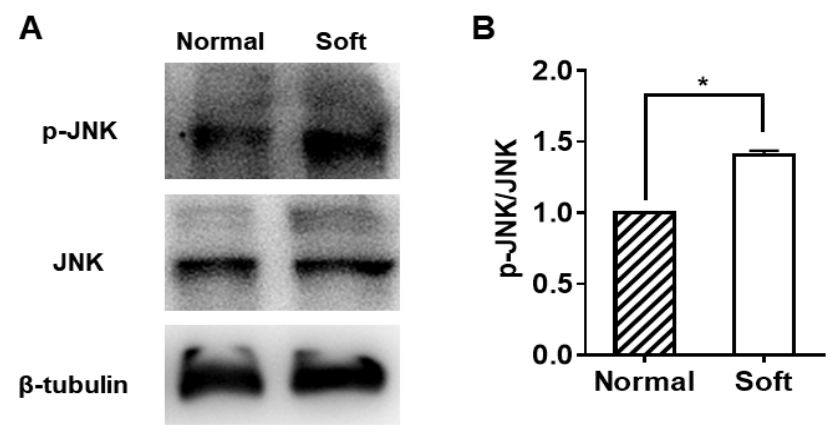

Figure 4. Phosphorylation of JNK in ECs. (A) After ECs reached complete confluence on different substrates, the phosphorylation of C-Jun N-terminal kinases (JNK) was studied by western blot analysis; (B) The statistics analysis of western blotting. The internal control in the western blot analysis here was $\beta$-tubulin.
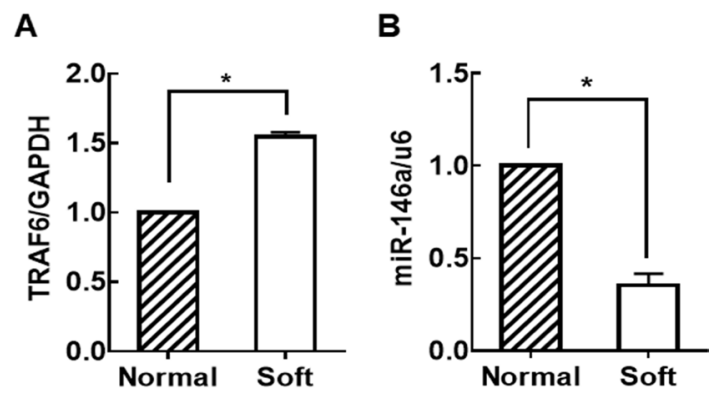

Figure 5. Expression of TRAF6 and miR-146a in ECs. After ECs reached complete confluence on soft or normal substrates, the expression of TRAF6 (A) and miR-146a (B) was analyzed by RT-qPCR. 
JNK signaling by soft substrate. Since it has been verified that miR-146a was a direct suppressor of TRAF6 [17] [18] [19], the expression of miR-146a was determined subsequently. Consistently, miR-146a was significantly less (0.4-fold) in ECs on soft rigidity substrate compared to $20 \mathrm{kPa}$ group (Figure 5(B)), indicating inhibition of miR-146a expression by soft ECM rigidity. These results implied that soft substrate promoted the phosphorylation of JNK via reducing miR-146a (targeting TRAF6) expression compared to normal substrate rigidity.

\section{Discussion and Conclusion}

It was reported that at the early stage, high fat diet resulted in decrease of vessel stiffness due to deposition of LDL and the lipid rich area of a vessel was $5.5 \pm 3.5$ $\mathrm{kPa}$ [7]. Thus we fabricated substrates with rigidity matching the stiffness of softened vessels and normal vessels. Surprisingly, the results pointed out that soft ECM rigidity greatly elevated the expression of pro-inflammatory cytokines in ECs. Further investigation found that down-regulation of miR-146a induced by soft ECM rigidity led to up-regulation of TRAF6 thus activating the inflammatory JNK signaling pathway.

TRAF6 is an important regulator in the MAPK signaling pathway. It was reported that TRAF6 regulates the expression of TGF- $\beta$-associated kinase 1 via regulating TAK-associated binding protein 1 , leading to phosphorylation of MAP kinase kinase 7 (MKK7) [20]. Subsequent phosphorylation of JNK induced by phosphorylation of MKK7 resulted in activation of transcription factors, initiating transcription of pro-inflammatory gene expression eventually. Furthermore, the expression of miR-146a, a suppressor of TRAF6, was shown to be down-regulated by substrate rigidity. Thus, our finding indicated that substrate rigidity can regulate the inflammatory response of endothelial cells via miR-146a expression. However, whether there are any other microRNAs involved requires further investigation.

Besides, our study has revealed a new possible physiological function of LDL deposition. Although the biochemical properties of LDL on cell functions have been studied intensively [21], whether the physical properties of LDL impacted the functions of cells remained poorly understood. Our study clearly indicated that changes of physical microenvironment of vessel wall, probably resulted from LDL depositing, induced inflammatory response of ECs. In addition, since these results implied strong regulation of substrate rigidity on ECs inflammatory response, substrate rigidity should be taken into serious consideration in the designing of vessel-on-a-chip system.

In conclusion, the results reported here, from a novel point of view, provided useful information for the successful designing of vessel-on-a-chip system as well as for the pathogenesis of atherosclerosis.

\section{Acknowledgements}

The human umbilical vein endothelial cell line is a gift from Prof. Guoyuan 
Yang, Shanghai Jiao Tong University. The work described in this paper was supported by the National Key Research and Development Program of China (2016YFC1100202), the Multidisciplinary Research Foundation of Shanghai Jiao Tong University (YG2017MS20) and Key Laboratory open project of Shanghai municipality (2016SGK-001).

\section{Conflicts of Interest}

The authors declare no conflicts of interest regarding the publication of this paper.

\section{References}

[1] Weber, C. and Noels, H. (2011) Atherosclerosis: Current Pathogenesis and Therapeutic Options. Nat Med, 17, 1410-1422. https://doi.org/10.1038/nm.2538

[2] Torrado, J., Buckley, L., Duran, A., Trujillo, P., Toldo, S., Valle, R.J., et al. (2018) Restenosis, Stent Thrombosis, and Bleeding Complications: Navigating between Scylla and Charybdis. J Am Coll Cardiol, 71, 1676-1695. https://doi.org/10.1016/j.jacc.2018.02.023

[3] Wang, L., Luo, J., Li, B., Tian, X.Y., Chen, L., Huang, Y., et al. (2016) Integrin-YAP/TAZ-JNK Cascade Mediates Atheroprotective Effect of Unidirectional Shear Flow. Nature, 540, 579. https://doi.org/10.1038/nature20602

[4] Wang, K., Yeh, Y., Phu, N., Limqueco, E., Lopez, J., Thorossian, S., et al. (2016) Flow-Dependent YAP/TAZ Activities Regulate Endothelial Phenotypes and Atherosclerosis. P Natl Acad Sci Usa, 113, 11525-11530. https://doi.org/10.1073/pnas.1613121113

[5] Gupta, P., Kumar, M., Bhardwaj, N., Kumar, J.P., Krishnamurthy, C.S., Nandi, S.K., et al. (2016) Mimicking Form and Function of Native Small Diameter Vascular Conduits Using Mulberry and Non-Mulberry Patterned Silk Films. ACS Appl Mater Interfaces, 8, 15874-15888. https://doi.org/10.1021/acsami.6b00783

[6] Matsumoto, T., Abe, H., Ohashi, T., Kato, Y. and Sato, M. (2002) Local Elastic Modulus of Atherosclerotic Lesions of Rabbit Thoracic Aortas Measured by Pipette Aspiration Method. Physiol Meas, 23, 635-648. https://doi.org/10.1088/0967-3334/23/4/304

[7] Tracqui, P., Broisat, A., Toczek, J., Mesnier, N., Ohayon, J. and Riou, L. (2011) Mapping Elasticity Moduli of Atherosclerotic Plaque in Situ via Atomic Force Microscopy. J Struct Biol, 174, 115-123. https://doi.org/10.1016/j.jsb.2011.01.010

[8] Yeung, T., Georges, P.C., Flanagan, L.A., Marg, B., Ortiz, M., Funaki, M., et al. (2005) Effects of Substrate Stiffness on Cell Morphology, Cytoskeletal Structure, and Adhesion. Cell Motility and the Cytoskeleton, 60, 24-34. https://doi.org/10.1002/cm.20041

[9] Aragona, M., Panciera, T., Manfrin, A., Giulitti, S., Michielin, F., Elvassore, N., et al. (2013) A Mechanical Checkpoint Controls Multicellular Growth through YAP/TAZ Regulation by Actin-Processing Factors. Cell, 154, 1047-1059.

https://doi.org/10.1016/j.cell.2013.07.042

[10] Ronaldson-Bouchard, K. and Vunjak-Novakovic, G. (2018) Organs-on-a-Chip: A Fast Track for Engineered Human Tissues in Drug Development. Cell Stem Cell, 22, 310-324. https://doi.org/10.1016/j.stem.2018.02.011

[11] Nie, J., Gao, Q., Wang, Y., Zeng, J., Zhao, H., Sun, Y., et al. (2018) Vessel-on-a-Chip 
with Hydrogel-Based Microfluidics. Small, 14, Article ID: e1802368. https://doi.org/10.1002/smll.201802368

[12] Li, Y., Zhu, K., Liu, X. and Zhang, Y.S. (2018) Blood-Vessel-on-a-Chip Platforms for Evaluating Nanoparticle Drug Delivery Systems. Curr Drug Metab, 19, 100-109. https://doi.org/10.2174/1389200218666170925114636

[13] Caballero, D., Blackburn, S.M., de Pablo, M., Samitier, J. and Albertazzi, L. (2017) Tumour-Vessel-on-a-Chip Models for Drug Delivery. Lab Chip, 17, 3760-3771. https://doi.org/10.1039/C7LC00574A

[14] Tse, J.R. and Engler, A.J. (2010) Preparation of Hydrogel Substrates with Tunable Mechanical Properties. Current Protocols in Cell Biology, 47, 10-16. https://doi.org/10.1002/0471143030.cb1016s47

[15] Luo, X., Li, H., Ma, L., Zhou, J., Guo, X., Woo, S.L., et al. (2018) Expression of STING Is Increased in Liver Tissues from Patients with NAFLD and Promotes Macrophage-Mediated Hepatic Inflammation and Fibrosis in Mice. Gastroenterology, 155, 1971-1984. https://doi.org/10.1053/j.gastro.2018.09.010

[16] Gulen, M.F., Kang, Z., Bulek, K., Youzhong, W., Kim, T.W., Chen, Y., et al. (2010) The Receptor SIGIRR Suppresses Th17 Cell Proliferation via Inhibition of the Interleukin-1 Receptor Pathway and mTOR Kinase Activation. Immunity, 32, 54-66. https://doi.org/10.1016/j.immuni.2009.12.003

[17] Xiong, Y., Yan, Y. and Li, Y. (2018) Tripterine Alleviates LPS-Induced Inflammatory Injury by Up-Regulation of miR-146a in HaCaT Cells. Biomed Pharmacother, 105, 798-804. https://doi.org/10.1016/j.biopha.2018.05.008

[18] Lai, N.S., Yu, H.C., Tung, C.H., Huang, K.Y., Huang, H.B. and Lu, M.C. (2017) The Role of Aberrant Expression of T Cell miRNAs Affected by TNF-Alpha in the Immunopathogenesis of Rheumatoid Arthritis. Arthritis Res Ther, 19, 261. https://doi.org/10.1186/s13075-017-1465-Z

[19] Roos, J., Enlund, E., Funcke, J.B., Tews, D., Holzmann, K., Debatin, K.M., et al. (2016) miR-146a-Mediated Suppression of the Inflammatory Response in Human Adipocytes. Sci Rep, 6, Article ID: 38339. https://doi.org/10.1038/srep38339

[20] Sorrentino, A., Thakur, N., Grimsby, S., Marcusson, A., von Bulow, V., Schuster, N., et al. (2008) The Type I TGF-Beta Receptor Engages TRAF6 to Activate TAK1 in a Receptor Kinase-Independent Manner. Nat Cell Biol, 10, 1199-207. https://doi.org/10.1038/ncb1780

[21] Chatterjee, S., Bedja, D., Mishra, S., Amuzie, C., Avolio, A., Kass, D.A., et al. (2014) Inhibition of Glycosphingolipid Synthesis Ameliorates Atherosclerosis and Arterial Stiffness in Apolipoprotein E-/-Mice and Rabbits Fed a High-Fat and -Cholesterol Diet. Circulation, 129, 2403-2413. https://doi.org/10.1161/CIRCULATIONAHA.113.007559 